\title{
Hard Vaginal Capsule Dosage Form
}

National Cancer Institute

\section{Source}

National Cancer Institute. Hard Vaginal Capsule Dosage Form. NCI Thesaurus. Code C150007.

Solid sing le-dose preparation consisting of a hard capsule of a size and shape suited for vaginal use, containing a liquid or semi-solid formulation, intended for a local effect. 